\title{
Survey of chemotherapy-induced nausea and vomiting in patients with urothelial carcinoma
}

\author{
AKIRA YOSHIMI $^{1-3}$, YUNA SHIROMA ${ }^{1}$, MIKU IWATA ${ }^{1}$, MARIKO NAKAMURA $^{1-3}$, \\ AYA TORII-GOTO $^{1}$, HIROTAKE HIDA ${ }^{1,3}$, NORIKO TANAKA ${ }^{3}$, MASAYUKI MIYAZAKI ${ }^{1,3}$, \\ KIYOFUMI YAMADA ${ }^{3}$ and YUKIHIRO NODA ${ }^{1-3}$
}

\author{
${ }^{1}$ Division of Clinical Sciences and Neuropsychopharmacology, Faculty and Graduate School of Pharmacy, \\ Meijo University; ${ }^{2}$ Clinical OMICs and Translation Research Center, Meijo University, Nagoya, Aichi 468-8503; \\ ${ }^{3}$ Department of Neuropsychopharmacology and Hospital Pharmacy, Graduate School of Medicine, \\ Nagoya University, Nagoya, Aichi 466-8560, Japan
}

Received April 9, 2021; Accepted July 19, 2021

DOI: $10.3892 / \mathrm{mco} .2021 .2384$

\begin{abstract}
Chemotherapy-induced nausea and vomiting (CINV) can cause anorexia, weight loss and deterioration of patient quality of life. It is one of the most unpleasant adverse effects of chemotherapy treatment regimens. For the optimal treatment of gastrointestinal symptoms during urothelial carcinoma chemotherapy, the present study investigated the association between gastrointestinal symptoms and therapeutic effects of gemcitabine plus platinum [cisplatin (GC) or carboplatin (GCa)] therapies. The incidence and frequency of nausea/vomiting with GC split therapy (gemcitabine, $1,000 \mathrm{mg} / \mathrm{m}^{2}$ on days 1 and 8 ; split-dose cisplatin, $35 \mathrm{mg} / \mathrm{m}^{2}$ on days 1 and 8; 21-day schedule) and GCa therapy [gemcitabine, $750-1,000 \mathrm{mg} / \mathrm{m}^{2}$ on days 1,8 and 15 ; carboplatin, area under the blood concentration-time curve $=5 \mathrm{mg} \mathrm{min} / \mathrm{ml}$ (Calvert formula) on day $2 ; 28$-day schedule] were lower compared with those of GC therapy (gemcitabine, $1,000 \mathrm{mg} / \mathrm{m}^{2}$ on days 1,8 and 15 ; single-dose cisplatin $70 \mathrm{mg} / \mathrm{m}^{2}$ on day 2 ; 28-day schedule). However, no differences in therapeutic
\end{abstract}

Correspondence to: Professor Yukihiro Noda, Division of Clinical Sciences and Neuropsychopharmacology, Faculty and Graduate School of Pharmacy, Meijo University, 150 Yagotoyama, Tempaku-ku, Nagoya, Aichi 468-8503, Japan

E-mail: ynoda@meijo-u.ac.jp

Abbreviations: AUC, area under the blood concentration-time curve; $\mathrm{Ccr}$, creatinine clearance; CINV, chemotherapy-induced nausea and vomiting; CR, complete response; eGFR, estimated glomerular filtration rate; GC, gemcitabine plus cisplatin; GCa, gemcitabine plus carboplatin; NK1, neurokinin-1; PD, progressive disease; PR, partial response; $\mathrm{SD}$, stable disease

Key words: chemotherapy-induced nausea and vomiting, gemcitabine plus carboplatin therapy, gemcitabine plus single-dose cisplatin therapy, gemcitabine plus split-dose cisplatin therapy, urothelial carcinoma outcomes were observed among therapies. GCa therapy, regardless of renal function, and GC split therapy demonstrated significant increases compared with GC therapy in alleviating gastrointestinal symptoms associated with cancer chemotherapy in patients with urothelial carcinoma. Overall, these results suggested that split-dose cisplatin administration or the use of carboplatin instead of cisplatin may be useful in patients who experience CINV without compromising treatment effectiveness.

\section{Introduction}

Platinum-based antineoplastic drugs, such as cisplatin, are reported to be effective against several malignant tumors (1); however, they frequently induce nausea and vomiting (2). Chemotherapy-induced nausea and vomiting (CINV) can cause anorexia and weakness $(3,4)$ and is one of the most unpleasant subjective symptoms experienced by patients (5). CINV significantly reduces the quality of life of patients (3) and adversely affects treatment continuation for the underlying disease $(6,7)$. Therefore, measures to alleviate CINV are crucial for the effective and safe administration of cancer chemotherapy.

Several guidelines $(2,8-10)$ have consistently recommended a three-drug combination therapy, consisting of serotonin $5-\mathrm{HT}_{3}$ receptor antagonists, selective neurokinin-1 (NK1) receptor antagonists, and corticosteroids as antiemetics for combating anticancer agents with a high emetic risk $(\geq 90 \%$; cisplatin), as well as those with moderate emetic risk (30-90\%; carboplatin) (2).

Patients with metastatic or advanced urothelial carcinoma are treated with gemcitabine plus cisplatin (GC) therapy, in which a single dose of cisplatin is administered in combination with gemcitabine as the standard of care $(11,12)$. In contrast, patients with reduced kidney function are prescribed GC split therapy (split-dose of cisplatin) $(13,14)$ or GCa therapy (administration of carboplatin instead of cisplatin) $(15,16)$. To our knowledge, the incidence of CINV and the efficacy of chemotherapy for these alternate treatment regimens compared to 
standard GC therapy in patients with reduced renal function has not been investigated previously.

To elucidate which regimen presented the lowest risk of CINV without compromising effectiveness, in this study, we compared the prevalence of gastrointestinal symptoms, use of antiemetics, therapeutic responses, and survival rates in patients receiving GC therapy, GC split therapy, or GCa therapy for urothelial carcinoma.

\section{Materials and methods}

Patients and therapeutic regimens. Patients who were treated with (a) GC therapy, (b) GC split therapy, or (c) GCa therapy (see below and Table I) at the urology ward of Nagoya University Hospital between March 1, 2011, and March 31, 2017, were retrospectively evaluated. For each therapy, the following scenarios were excluded from the analysis: patients who discontinued the use of platinum preparations (cisplatin or carboplatin), cases in which nausea and vomiting during the 10-day observation period could not be clarified, and cases in which the use of opioids rendered it challenging to determine whether nausea and vomiting were induced by chemotherapy.

(a) GC therapy. The GC therapy regimen was administered for 28 days (4-week interval), with cisplatin $\left(70 \mathrm{mg} / \mathrm{m}^{2}\right)$ administered on treatment day 2 and gemcitabine $\left(1,000 \mathrm{mg} / \mathrm{m}^{2}\right)$ administered on treatment days 1,8 , and 15 (Table I). On the day of cisplatin administration, patients were pre-administered serotonin $5-\mathrm{HT}_{3}$ receptor antagonists (ramosetron, granisetron, or palonosetron), a corticosteroid (dexamethasone), and a selective NK1 receptor antagonist (aprepitant). Aprepitant was administered on treatment days $2(125 \mathrm{mg}$; before cisplatin administration), 3 (80 $\mathrm{mg}$ ), and 4 (80 $\mathrm{mg})$.

(b) GC split therapy. For GC split therapy, the treatment regimen was administered for 21 days (3-week interval), with cisplatin $\left(35 \mathrm{mg} / \mathrm{m}^{2}\right)$ and gemcitabine $\left(1,000 \mathrm{mg} / \mathrm{m}^{2}\right)$ administered on treatment days 1 and 8 (Table I). On the days of cisplatin administration, patients were pre-administered serotonin $5-\mathrm{HT}_{3}$ receptor antagonist and dexamethasone. Aprepitant was administered on treatment days $1(125 \mathrm{mg}$; before administration of cisplatin), 2 (80 mg), and 3 (80 mg).

(c) GCa therapy. GCa treatment was performed for 28 days (4-week interval) and consisted of carboplatin [area under the blood concentration-time curve $(\mathrm{AUC})=5 \mathrm{mg} \cdot \mathrm{min} / \mathrm{ml}]$ administered on treatment day 2 and gemcitabine $\left(750-1,000 \mathrm{mg} / \mathrm{m}^{2}\right)$ administered on treatment days 1,8 , and 15 (Table I). On the day of carboplatin administration, patients were pre-administered with a serotonin $5-\mathrm{HT}_{3}$ receptor antagonist and dexamethasone.

Assessment. In this study, a 10-day (treatment days 1 to 10 ) observation period was employed from the start date of each course (GC therapy, GC split therapy, or GCa therapy). The following parameters were investigated: (i) number of days with nausea and vomiting, (ii) prevalence of nausea and vomiting on each treatment day, (iii) administration of a serotonin $5-\mathrm{HT}_{3}$ receptor antagonist, (iv) total and additional use of antiemetics (average use count, total dose, and use date of serotonin $5-\mathrm{HT}_{3}$ receptor antagonists, aprepitant, dopamine $\mathrm{D}_{2}$ receptor antagonists), (v) therapeutic response [complete response (CR), partial response (PR), stable disease (SD), or progressive disease (PD)] based on the new response evaluation criteria in solid tumors (RECIST v.1.1) (17), and (vi) the duration of patient survival from the start date of each course of treatment to the date of the final follow-up observation or date of death.

Statistical analysis. Fisher's exact test was used to analyze categorical data related to sex, rate of incidence of nausea and vomiting events on each treatment day, use rate of each serotonin $5-\mathrm{HT}_{3}$ receptor antagonist, use rate of additional antiemetics and prevalence of patients with each therapeutic response. The Shapiro-Wilk test was used to determine normality, and Leven's test was used to assess the equality of variances. The Kruskal-Wallis test was used to compare median values of age, estimated glomerular filtration rate (eGFR), creatinine clearance (Ccr) and the number of days with nausea/vomiting per course among three groups, followed by post-hoc testing using the unpaired Mann-Whitney U test, with a Bonferroni-adjusted alpha level. The Cramér-von Mises test was used to assess survival rates. Statistical significance was set at $\mathrm{P}<0.05$. Data analysis, power analysis, and sample size calculations were performed using the $\mathrm{R}$ statistical software (version 3.6.2; R Foundation for Statistical Computing, Vienna, Austria).

\section{Results}

Patient characteristics. In total, 67 patients (48 men and 19 women) were included in this study. There were 43 patients (27 men, 16 women) in the GC therapy group, 9 patients (8 men, 1 woman) in the GC split therapy group, and 15 patients (13 men, 2 women) in the GCa group. There were no statistically significant differences in age or sex among the groups. However, eGFR and Ccr levels were significantly lower in the GC split and GCa therapy groups than in the GC therapy group $(\mathrm{P}<0.01)$, and the level of $\mathrm{Ccr}$ was also significantly lower in the GCa therapy group than in the GC split therapy group $(\mathrm{P}<0.05$; Table II).

Number of days with nausea/vomiting per course. The number of days with nausea/vomiting per course \pm standard deviation in the GC, GC split, and GCa therapy groups is shown in Fig. 1. We found that the number of days with nausea per course was significantly lower in the GC split and GCa therapy groups than in the GC therapy group $(\mathrm{P}<0.01, \mathrm{P}<0.05$, respectively; Fig. 1A). Although several patients experienced vomiting during the observation period in the GC and GCa therapy groups, there were no patients with vomiting episodes in the GC split therapy group (Fig. 1B).

Prevalence of nausea and vomiting on each treatment day. The prevalence of nausea on treatment days 4 to 7 was significantly lower in the GC split therapy group than in the GC therapy group (treatment days 4 and 6 : $\mathrm{P}<0.01$, treatment days 5 and 7: $\mathrm{P}<0.05$; Fig. 2A). On treatment days 3 and 4, prevalence of nausea/vomiting was significantly lower in the GCa therapy group than in the GC therapy group $(\mathrm{P}<0.01$; Fig. 2A). No differences in the prevalence of vomiting were observed among the treatment groups $(\mathrm{P}<0.05$; Fig. $2 \mathrm{~B})$. 


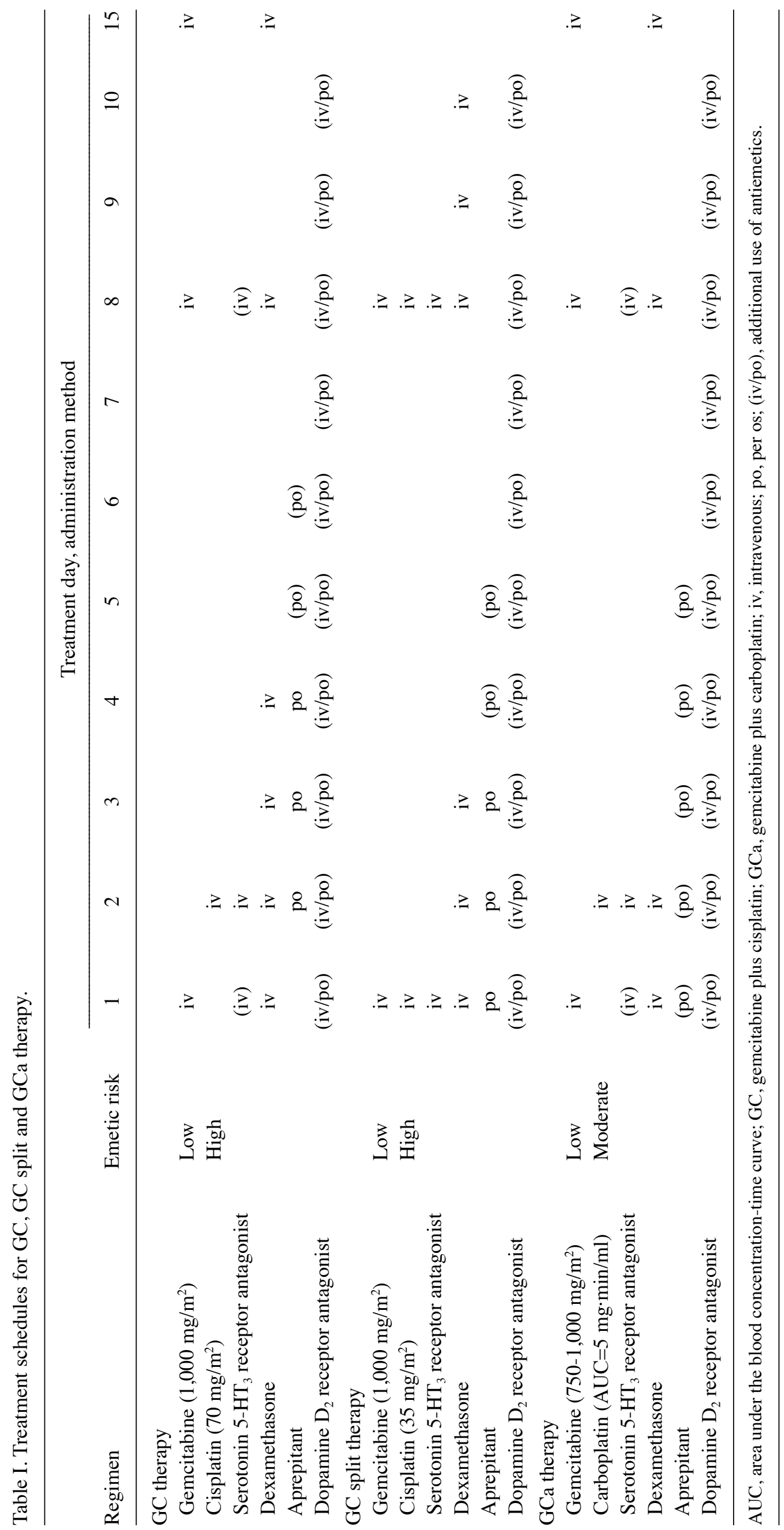


Table II. Patient characteristics.

\begin{tabular}{lccc}
\hline Characteristic & GC therapy & GC split therapy & GCa therapy \\
\hline Median age, years (range) & $67.0(36-80)$ & $67.0(49-76)$ & $73.0(57-84)$ \\
Sex, $\mathrm{n}(\%)$ & $27(62.8)$ & $8(88.9)$ & $13(86.7)$ \\
Men & $16(37.2)$ & $1(11.1)$ & $2(13.3)$ \\
Women & 72 & $38.4(31.8-50.6)^{\mathrm{a}}$ & 19 \\
Number of chemotherapy courses & $61.8(36.9-162.5)$ & $37.1(15.8-64.5)^{\mathrm{a}}$ \\
Median eGFR, ml/min/1.73 $\mathrm{m}^{2}$ (range) & $57.6(31.5-153.8)$ & $46.0(34.5-62.3)^{\mathrm{a}}$ & $41.2(18.1-70.6)^{\mathrm{a}, \mathrm{b}}$ \\
Median Ccr, ml/min (range) & & & \\
\hline
\end{tabular}

Ccr, creatinine clearance; eGFR, estimated glomerular filtration rate; GC, gemcitabine plus cisplatin; GCa, gemcitabine plus carboplatin. ${ }^{\mathrm{a}} \mathrm{P}<0.01$ vs. GC therapy and ${ }^{\mathrm{b}} \mathrm{P}<0.05$ vs. GC split therapy.

A

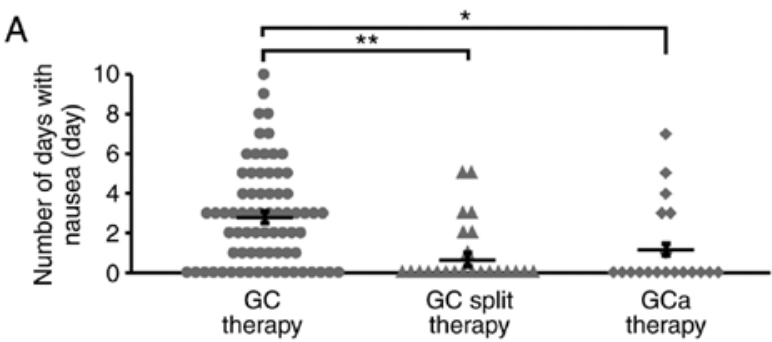

B

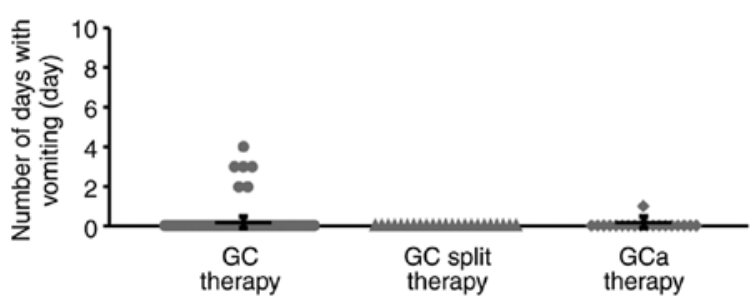

Figure 1. Number of days with nausea/vomiting per course in the GC, GC split and GCa therapy groups. Mean number of days with (A) nausea and (B) vomiting per course is shown as a scatter plot with mean \pm standard error of the mean for the GC therapy group ( 72 courses), GC split therapy group (23 courses) and GCa therapy group ( 19 courses). ${ }^{*} \mathrm{P}<0.05,{ }^{* *} \mathrm{P}<0.01 \mathrm{vs}$. GC therapy group. GC, gemcitabine plus cisplatin; GCa, gemcitabine plus carboplatin.

Use rate of each serotonin 5-HT $\mathrm{H}_{3}$ receptor antagonist. The use rates of serotonin 5- $\mathrm{HT}_{3}$ receptor antagonists (ramosetron, granisetron, or palonosetron) in each therapy group are shown in Fig. 3 . A significant difference was observed in the use rate between the GC split therapy and the GC therapy groups $(\mathrm{P}<0.05$; Fig. 3). In particular, a second-generation serotonin $5-\mathrm{HT}_{3}$ receptor antagonist (palonosetron) was significantly lower in the GC split therapy group ( $\mathrm{P}<0.05 ;$ Fig. 3). No differences were observed between the $\mathrm{GCa}$ and GC therapy groups or the GC split therapy group.

Total and additional use of antiemetics. The total and additional use of antiemetics is summarized in Table III. The total dose of antiemetics during the observation period was significantly lower in the GC split therapy group than in the GC and GCa therapy groups $(\mathrm{P}<0.01)$. The metoclopramide dose was significantly lower in the GC split therapy group than in the GC therapy group $(\mathrm{P}<0.05)$. Aprepitant dose was significantly lower in the GCa therapy group than in the GC and GC split therapy groups $(\mathrm{P}<0.01)$.
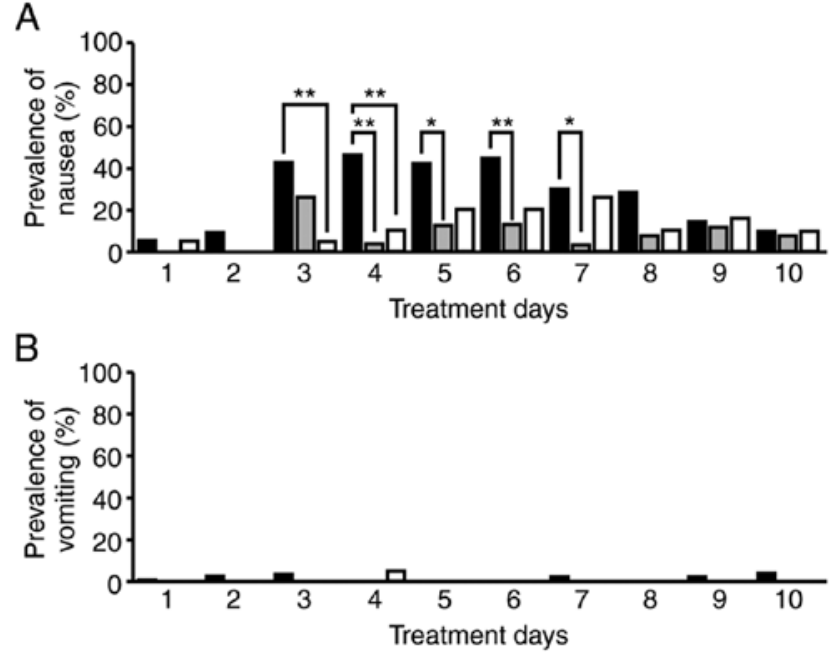

Figure 2. Prevalence of nausea/vomiting on each treatment day in the GC, GC split and GCa therapy groups. Prevalence of (A) nausea and (B) vomiting between treatment days 1-10 is presented for the GC therapy group (black; 72 courses), GC split therapy group (gray; 23 courses) and GCa therapy group (white; 19 courses). ${ }^{*} \mathrm{P}<0.05,{ }^{* *} \mathrm{P}<0.01$ vs. GC therapy group. GC, gemcitabine plus cisplatin; GCa, gemcitabine plus carboplatin.

The use rates of additional antiemetics in the GC, GC split, and GCa therapy groups were $70.8 \%$ (51/72 courses), $39.1 \%$ (9/23 courses), and 78.9\% (15/19 courses), respectively. The use rate was significantly lower in the GC split therapy group than in the GC and GCa therapy groups $(\mathrm{P}<0.05)$. Considering the dose of additional antiemetics during the observation period, aprepitant dose was significantly lower in the GC split therapy group than in the GC therapy group $(\mathrm{P}<0.01)$, whereas it was significantly higher in the GCa therapy group than in the GC and GC split therapy groups $(\mathrm{P}<0.01$; Table III).

Therapeutic response and survival rates. No differences were observed in the proportion of each therapeutic response in the GC therapy group [CR: 0\% (0/34 patients), PR: $8.8 \%$ (3/34 patients), SD: 50.0\% (17/34), PD: $41.2 \%$ (14/34 patients)], GC split therapy group [CR: $0 \%$ (0/4 patients), PR: $25.0 \%$ (1/4 patients), SD: $75.0 \%$ (3/4 patients), PD: $0.0 \%$ (0/4 patients)], and GCa therapy group [CR: $0 \%$ (0/6 patients), PR: $16.7 \%$ (1/6 patients), SD: $66.7 \%$ (4/6 patients), PD: $16.7 \%$ 


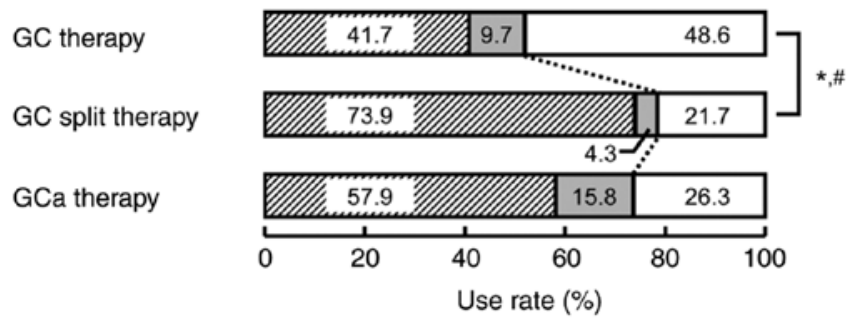

Figure 3. Use rate of each serotonin $5-\mathrm{HT}_{3}$ receptor antagonist in the GC, GC split and the GCa therapy groups. Use rates of ramosetron (diagonal lines), granisetron (gray), and palonosetron (white) are shown for the GC therapy group (72 courses), GC split therapy group (23 courses) and GCa therapy group (19 courses). "P<0.05, comparison of the rate of each serotonin $5-\mathrm{HT}_{3}$ receptor antagonist; ${ }^{\prime} \mathrm{P}<0.05$, comparison of the rate between the first-generation (ramosetron and granisetron) and the second-generation [serotonin 5- $\mathrm{HT}_{3}$ receptor antagonist (palonosetron)] vs. GC therapy group. $\mathrm{GC}$, gemcitabine plus cisplatin; $\mathrm{GCa}$, gemcitabine plus carboplatin.

(1/6 patients)] $(\mathrm{P}>0.05)$. No differences in the duration of survival or survival rates were observed among the treatment groups ( $\mathrm{P}>0.05$; Fig. 4).

Power analysis and sample size calculation. This study had a power of 0.42-0.59 to detect a medium effect (18) and could not obtain the estimated sample size $(n=53-108)$.

\section{Discussion}

This study compared the prevalence of gastrointestinal symptoms, use of antiemetics, therapeutic responses, and survival rates in patients receiving GC therapy, GC split therapy, or GCa therapy for urothelial carcinoma. Although CINV in the acute phase (within the first $24 \mathrm{~h}$ after chemotherapy) was well-controlled in all therapy groups, patients receiving GC therapy showed a higher incidence of vomiting in the delayed phase ( $>24 \mathrm{~h}$ after chemotherapy) than the other groups. In the GC split therapy group, there were no vomiting episodes, and the number of days with nausea per course and the prevalence of nausea on treatment days 4 to 7 were significantly lower than those observed in the GC therapy group. This suggests that low-dose cisplatin administration can suppress the emergence of delayed nausea. In the GCa therapy group, the number of days with nausea per course was significantly lower, and the prevalence of nausea on treatment days 3 and 4 was significantly lower than that observed in the GC therapy group. These results can be explained by the fact that carboplatin is a platinum-based antineoplastic drug with moderate emetic risk (2).

A second-generation serotonin $5-\mathrm{HT}_{3}$ receptor antagonist, palonosetron, is effective against both the acute and delayed phases of CINV (19). Among the serotonin 5- $\mathrm{HT}_{3}$ receptor antagonists used in this survey, the use rate of palonosetron in the GC split and GCa therapy groups was lower than that in the GC therapy group. This could be attributed to the fewer number of days with nausea per course, as well as the lower prevalence of nausea on each treatment day in both the GC split and GCa therapy groups than in the GC therapy group. Therefore, our findings suggest that both GC split therapy and GCa therapy can reduce the prevalence of acute and delayed CINV, regardless of palonosetron administration. The GC

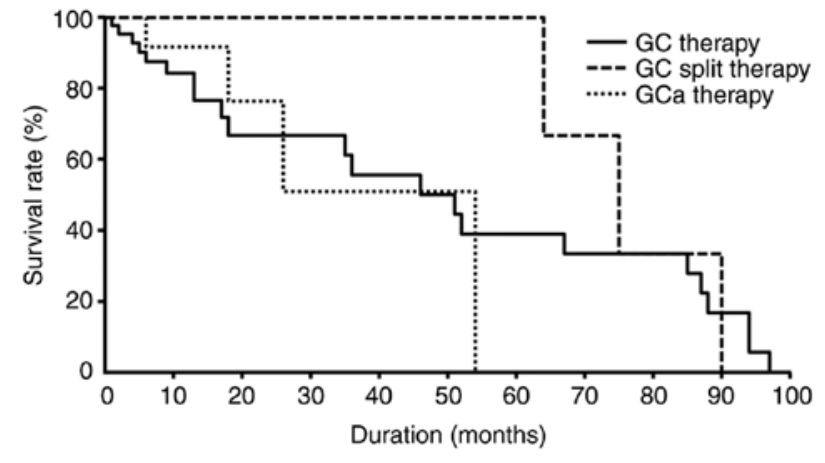

Figure 4. Survival curves for the GC, GC split and GCa therapy groups. Survival rates and duration of survival are shown for the GC therapy group (43 patients), GC split therapy group ( 9 patients) and GCa therapy group (15 patients). GC, gemcitabine plus cisplatin; GCa, gemcitabine plus carboplatin.

split therapy group presented a significantly lower administration rate and aprepitant dose of additional antiemetics than the GC therapy and GCa therapy groups. This could be attributed to several factors. First, GC split therapy has a lower risk of CINV than GC or GCa therapy. Second, all patients receiving GC therapy and GC split therapy, who were administered cisplatin (a high emetic risk drug), were also administered a three-drug combination antiemetic therapy according to the treatment schedules. Third, patients receiving GCa therapy, using carboplatin, were additionally administered aprepitant, which was not listed in the treatment schedule. When we compared the need for antiemetic use during the observation period, the aprepitant dose was significantly lower in the GCa therapy group than in the GC and GC split therapy groups. Moreover, the frequency of additional use of serotonin 5- $\mathrm{HT}_{3}$ receptor antagonist was lower in aprepitant users (16.7\%) than in non-users $(61.5 \%)$. GCa therapy can reduce both early and late CINV with the minimum requirement of aprepitant use, and aprepitant use may result in additional antiemetic effects. Furthermore, although the treatment duration for the GC split therapy was shorter (21 days) than that for a single course of GC therapy (28 days), CINV events were adequately controlled with the three-drug antiemetic therapy. Therefore, if the dose of cisplatin administered per course was the same, split-dose treatments may be more effective in mitigating CINV onset than single-dose treatments.

Cisplatin-based treatment regimens are used as first-line therapies for metastatic or advanced urothelial carcinoma; however, the use of cisplatin has been associated with renal dysfunction, deterioration of the general condition, and medical complications in $40-50 \%$ of patients (20). Carboplatin or split-dose administration of cisplatin is employed as an alternative platinum formulation in patients presenting with these adverse events (13-16). In this study, we found that eGFR and Ccr levels were significantly lower in patients receiving GC split therapy and GCa therapy than in those receiving GC therapy, suggesting that GC split therapy and GCa therapy can be prioritized in patients with reduced kidney function. It has been suggested that adverse events are less common in regimens that use carboplatin instead of cisplatin (21), although the therapeutic efficacy of cisplatin is significantly inferior to that of carboplatin (22). However, only a few reports have presented direct comparisons between GC, GC split, and GCa therapies $(16,23)$. 
Table III. Antiemetics.

A, Total use of antiemetics (included in regimen and additional use).

\begin{tabular}{|c|c|c|c|c|}
\hline Antiemetics & Regimen & $\mathrm{N}^{\mathrm{a}}$ & $\begin{array}{c}\text { Mean count } \pm \mathrm{SD} \\
\text { (range) }\end{array}$ & $\begin{array}{c}\text { Mean dose, } \\
\mathrm{mg} / \text { course } \pm \mathrm{SD}(\text { range })\end{array}$ \\
\hline \multirow[t]{3}{*}{ Granisetron } & $\mathrm{GC}$ & 7 & $1.14 \pm 0.38(1-2)$ & $2.00 \pm 1.00(1-3)$ \\
\hline & GC split & 1 & $1.00 \pm 0.00(1)$ & $3.00 \pm 0.00(3)$ \\
\hline & $\mathrm{GCa}$ & 3 & $1.00 \pm 0.00$ & $3.00 \pm 0.00(3)$ \\
\hline \multirow[t]{3}{*}{ Palonosetron } & $\mathrm{GC}$ & 35 & $1.00 \pm 0.00$ & $0.75 \pm 0.00(0.75)$ \\
\hline & GC split & 5 & $1.00 \pm 0.00$ & $0.75 \pm 0.00(0.75)$ \\
\hline & $\mathrm{GCa}$ & 5 & $1.00 \pm 0.00$ & $0.75 \pm 0.00(0.75)$ \\
\hline \multirow[t]{3}{*}{ Ramosetron } & GC & 30 & $2.23 \pm 0.94(1-3)$ & $0.67 \pm 0.28(0.3-0.9)$ \\
\hline & GC split & 17 & $1.00 \pm 0.00(1)$ & $0.30 \pm 0.00(0.3)^{\mathrm{d}}$ \\
\hline & $\mathrm{GCa}$ & 11 & $2.55 \pm 0.82(1-3)$ & $0.74 \pm 0.28(0.3-0.9)^{\mathrm{e}}$ \\
\hline Domperidone & GC & 72 & $0.15 \pm 0.82(0-6)$ & $3.61 \pm 22.35(0-180)$ \\
\hline \multirow[t]{3}{*}{ Metoclopramide } & GC & 72 & $4.04 \pm 8.96(0-31)$ & $20.97 \pm 45.48(0-160)$ \\
\hline & GC split & 23 & $0.06 \pm 0.24(0-1)$ & $0.29 \pm 1.21(0-5)^{\mathrm{c}}$ \\
\hline & $\mathrm{GCa}$ & 19 & $2.16 \pm 4.89(0-15)$ & $11.05 \pm 24.47(0-75)$ \\
\hline \multirow[t]{3}{*}{ Aprepitant } & GC & 72 & $3.57 \pm 0.90(3-5)$ & $330.56 \pm 72.09(285-445)$ \\
\hline & GC split & 23 & $3.35 \pm 0.49(3-4)$ & $313.24 \pm 39.41(285-365)$ \\
\hline & $\mathrm{GCa}$ & 19 & $0.95 \pm 1.43(0-3)$ & $90.00 \pm 136.11(0-285)^{\mathrm{d}, \mathrm{e}}$ \\
\hline
\end{tabular}

B, Additional use of antiemetics (not included in regimen).

\begin{tabular}{|c|c|c|c|c|c|}
\hline Antiemetics & Regimen & $\mathrm{N}^{\mathrm{b}}$ & $\begin{array}{l}\text { Mean count } \pm \text { SD } \\
\quad(\text { range })\end{array}$ & $\begin{array}{c}\text { Mean dose, } \\
\mathrm{mg} / \text { course } \pm \mathrm{SD} \text { (range) }\end{array}$ & Day administered $(\mathrm{N})$ \\
\hline Granisetron & GC & 1 & $1.00 \pm 0.00(1)$ & $1.00 \pm 0.00(1)$ & Day $1(1)$ \\
\hline \multirow[t]{2}{*}{ Ramosetron } & GC & 20 & $1.85 \pm 0.37(1-2)$ & $0.56 \pm 0.11(0.3-0.6)$ & Day 1 (20), day 8 (17) \\
\hline & $\mathrm{GCa}$ & 9 & $1.89 \pm 0.33(1-2)$ & $0.57 \pm 0.10(0.3-0.6)$ & Day 1 (9), day 8 (8) \\
\hline Domperidone & $\mathrm{GC}$ & 3 & $3.67 \pm 2.08(2-6)$ & $86.67 \pm 83.27(20-180)$ & $\begin{array}{l}\text { Day } 3(1) \text {, day } 4(2) \text {, day } 5(3) \text {, } \\
\text { day } 6(2) \text {, day } 7 \text { (1) }\end{array}$ \\
\hline \multirow[t]{3}{*}{ Metoclopramide } & GC & 29 & $10.03 \pm 11.88(1-30)$ & $52.07 \pm 59.72(5-150)$ & $\begin{array}{l}\text { Day } 1(7) \text {, day } 2(8) \text {, day } 3(12) \text {, } \\
\text { day } 4(13) \text {, day } 5(15) \text {, day } 6(16) \text {, } \\
\text { day } 7(15) \text {, day } 8(15) \text {, day } 9(10) \text {, } \\
\text { day } 10(9)\end{array}$ \\
\hline & GC split & 1 & $1.00 \pm 0.00$ & $5.00 \pm 0.00$ & Day $9(1)$ \\
\hline & $\mathrm{GCa}$ & 4 & $10.25 \pm 5.74(2-15)$ & $52.50 \pm 26.30(15-75)$ & $\begin{array}{l}\text { Day } 3(1) \text {, day } 4(1) \text {, day } 5(2) \text {, } \\
\text { day } 6(3) \text {, day } 7(3) \text {, day } 8(2) \text {, } \\
\text { day } 9(3) \text {, day } 10(2)\end{array}$ \\
\hline \multirow[t]{3}{*}{ Aprepitant } & $\mathrm{GC}$ & 21 & $1.95 \pm 0.22(1-2)$ & $156.19 \pm 17.46(80-160)$ & Day 5 (21), day 6 (20) \\
\hline & GC split & 9 & $1.00 \pm 0.00(1)$ & $80.00 \pm 0.00(80)^{\mathrm{d}}$ & Day $4(9)$ \\
\hline & $\mathrm{GCa}$ & 6 & $3.00 \pm 0.00(3)$ & $285.00 \pm 0.00(285)^{\mathrm{d}, \mathrm{e}}$ & Day 2 (6), day 3 (6), day 4 (6) \\
\hline
\end{tabular}

$\mathrm{SD}$, standard deviation; N, the number of courses; GC, gemcitabine plus cisplatin; GCa, gemcitabine plus carboplatin; date, treatment day during observation period (from day 1-10). ${ }^{a}$ Number of serotonin 5- $\mathrm{HT}_{3}$ receptor antagonists (granisetron, palonosetron and ramosetron) indicates courses of each serotonin 5- $\mathrm{HT}_{3}$ receptor antagonist users receiving each chemotherapy. The number of dopamine $\mathrm{D}_{2}$ receptor antagonists (domperidone and metoclopramide) and neurokinin-1 receptor antagonist (aprepitant) indicates all courses receiving each chemotherapy. ${ }^{\mathrm{b}}$ Additional user of each antiemetics. ${ }^{\mathrm{c}} \mathrm{P}<0.05,{ }^{\mathrm{d}} \mathrm{P}<0.01$ vs GC therapy and ${ }^{\mathrm{e}} \mathrm{P}<0.01$ vs GC split therapy.

Results from our preliminary survey indicated that there were no significant differences in the therapeutic effects and survival rates among the three groups. Therefore, the selection of GC split therapy or GCa therapy, instead of GC therapy, may reduce CINV incidence without adversely affecting the therapeutic effects and survival rates in patients with urothelial carcinoma. 
This study was limited by its retrospective design based on electronic medical records and a small sample size compared to the estimated ideal sample size $(n=53-108)$, which may induce biases owing to insufficient statistical power (0.42-0.59 to detect a medium effect). Additionally, it is possible that the patients' memories and medical staff records were inaccurate, resulting in recall bias. As there was probably some information that was not recorded in the medical records, it should be noted that the occurrence of nausea/vomiting may have been underestimated. Furthermore, we did not examine the relationship between patient-related factors (e.g., age, sex, and history of alcohol intake) or genetic risk factors $(6,24,25)$ and the incidence of CINV. Therefore, these results should be interpreted with caution.

In conclusion, GC split therapy and GCa therapy were superior to GC therapy in reducing the incidence of CINV in patients with urothelial carcinoma. In particular, GCa therapy is likely to be superior to GC and GC split therapy because carboplatin can be administered regardless of renal function and has few side effects such as nausea and vomiting.

Although further investigations on the therapeutic effects and other adverse events are required, split-dose cisplatin administration or the use of carboplatin instead of cisplatin may be useful in patients who experience CINV without compromising treatment effectiveness.

\section{Acknowledgements}

Not applicable.

\section{Funding}

This study was supported by a grant from The Encouragement of Scientific Research, Promoted Research Center Subsidy by Meijo University Research Institute.

\section{Availability of data and materials}

The datasets used and/or analyzed during the current study are available from the corresponding author on reasonable request.

\section{Authors' contributions}

AY, MM, KY and YN conceived and designed the current study. AY, YS, MI, MN, ATG, HH, NT and MM acquired the data. AY, YS, MI, MN, ATG and YN analyzed and interpreted the data. AY, YS and MI performed statistical analysis. AY and YS drafted the manuscript. AY and YN supervised the study and confirmed the authenticity of all the raw data. YN critically revised the manuscript for important intellectual content. All authors have read and approved the final version of the manuscript.

\section{Ethics approval and consent to participate}

This survey was conducted in accordance with the Declaration of Helsinki and the Ethical Guidelines for Medical and Health Research Involving Human Subjects (26). This study was approved by the Ethics Committee of Nagoya University
Graduate School of Medicine (approval no. 2016-0539-3), and written informed consent was waived because of the retrospective design. To protect personal information, all data obtained were handled after anonymization.

\section{Patient consent for publication}

Not applicable.

\section{Competing interests}

The authors declare that they have no competing interests.

\section{References}

1. Dasari S and Tchounwou PB: Cisplatin in cancer therapy: Molecular mechanisms of action. Eur J Pharmacol 740: 364-378, 2014.

2. Razvi Y, Chan S, McFarlane T, McKenzie E, Zaki P, DeAngelis C, Pidduck W, Bushehri A, Chow E and Jerzak KJ: ASCO, NCCN, MASCC/ESMO: A comparison of antiemetic guidelines for the treatment of chemotherapy-induced nausea and vomiting in adult patients. Support Care Cancer 27: 87-95, 2019.

3. Bloechl-Daum B, Deuson RR, Mavros P, Hansen M and Herrstedt J: Delayed nausea and vomiting continue to reduce patients' quality of life after highly and moderately emetogenic chemotherapy despite antiemetic treatment. J Clin Oncol 24: 4472-4478, 2006.

4. Navari RM: Management of chemotherapy-induced nausea and vomiting: Focus on newer agents and new uses for older agents. Drugs 73: 249-262, 2013.

5. Coates A, Abraham S, Kaye SB, Sowerbutts T, Frewin C, Fox RM and Tattersall $\mathrm{MH}$ : On the receiving end-patient perception of the side-effects of cancer chemotherapy. Eur J Cancer Clin Oncol 19: 203-208, 1983.

6. Hesketh PJ: Chemotherapy-induced nausea and vomiting. N Engl J Med 358: 2482-2494, 2008.

7. Van Laar ES, Desai JM and Jatoi A: Professional educational needs for chemotherapy-induced nausea and vomiting (CINV): Multinational survey results from 2388 health care providers. Support Care Cancer 23: 151-157, 2015.

8. Hesketh PJ, Kris MG, Basch E, Bohlke K, Barbour SY Clark-Snow RA, Danso MA, Dennis K, Dupuis LL, Dusetzina SB, et al: Antiemetics: American society of clinical oncology clinical practice guideline update. J Clin Oncol 35: 3240-3261, 2017.

9. Berger MJ, Ettinger DS, Aston J, Barbour S, Bergsbaken J Bierman PJ, Brandt D, Dolan DE, Ellis G, Kim EJ, et al: NCCN guidelines insights: Antiemesis, version 2.2017. J Natl Compr Canc Netw 15: 883-893, 2017.

10. Roila F, Molassiotis A, Herrstedt J, Aapro M, Gralla RJ, Bruera E, Clark-Snow RA, Dupuis LL, Einhorn LH, Feyer P, et al: 2016 MASCC and ESMO guideline update for the prevention of chemotherapy- and radiotherapy-induced nausea and vomiting and of nausea and vomiting in advanced cancer patients. Ann Oncol 27 (Suppl 5): v119-v133, 2016.

11. von der Maase H, Sengelov L, Roberts JT, Ricci S, Dogliotti L, Oliver T, Moore MJ, Zimmermann A and Arning M: Long-term survival results of a randomized trial comparing gemcitabine plus cisplatin, with methotrexate, vinblastine, doxorubicin, plus cisplatin in patients with bladder cancer. J Clin Oncol 23: 4602-4608, 2005.

12. Bellmunt J, von der Maase H, Mead GM, Skoneczna I, De Santis M, Daugaard G, Boehle A, Chevreau C, Paz-Ares L, Laufman LR, et al: Randomized phase III study comparing paclitaxel/cisplatin/gemcitabine and gemcitabine/cisplatin in patients with locally advanced or metastatic urothelial cancer without prior systemic therapy: EORTC intergroup study 30987. J Clin Oncol 30: 1107-1113, 2012.

13. Hussain SA, Stocken DD, Riley P, Palmer DH, Peake DR, Geh JI, Spooner D and James ND: A phase I/II study of gemcitabine and fractionated cisplatin in an outpatient setting using a 21-day schedule in patients with advanced and metastatic bladder cancer. Br J Cancer 91: 844-849, 2004. 
14. Kim YR, Lee JL, You D, Jeong IG, Song C, Hong B, Hong JH and Ahn H: Gemcitabine plus split-dose cisplatin could be a promising alternative to gemcitabine plus carboplatin for cisplatin-unfit patients with advanced urothelial carcinoma. Cancer Chemother Pharmacol 76: 141-153, 2015.

15. De Santis M, Bellmunt J, Mead G, Kerst JM, Leahy M, Maroto P, Gil T, Marreaud S, Daugaard G, Skoneczna I, et al: Randomized phase II/III trial assessing gemcitabine/carboplatin and methotrexate/carboplatin/vinblastine in patients with advanced urothelial cancer who are unfit for cisplatin-based chemotherapy: EORTC study 30986. J Clin Oncol 30: 191-199, 2012.

16. Einstein DJ and Sonpavde G: Treatment approaches for cisplatin-ineligible patients with invasive bladder cancer. Curr Treat Options Oncol 20: 12, 2019.

17. Eisenhauer EA, Therasse P, Bogaerts J, Schwartz LH, Sargent D, Ford R, Dancey J, Arbuck S, Gwyther S, Mooney M, et al: New response evaluation criteria in solid tumours: Revised RECIST guideline (version 1.1). Eur J Cancer 45: 228-247, 2009.

18. Cohen J: A power primer. Psychol Bull 112: 155-159, 1992.

19. Saito M, Aogi K, Sekine I, Yoshizawa H, Yanagita Y, Sakai H, Inoue K, Kitagawa C, Ogura T and Mitsuhashi S: Palonosetron plus dexamethasone versus granisetron plus dexamethasone for prevention of nausea and vomiting during chemotherapy: A double-blind, double-dummy, randomised, comparative phase III trial. Lancet Oncol 10: 115-124, 2009.

20. Morales-Barrera R, Bellmunt J, Suárez C, Valverde C, Guix M, Serrano C, Gallén M and Carles J: Cisplatin and gemcitabine administered every two weeks in patients with locally advanced or metastatic urothelial carcinoma and impaired renal function. Eur J Cancer 48: 1816-1821, 2012.
21. Sakaeda T, Kadoyama K and Okuno Y: Adverse event profiles of platinum agents: Data mining of the public version of the FDA adverse event reporting system, AERS, and reproducibility of clinical observations. Int J Med Sci 8: 487-491, 2011.

22. de Vos FY and de Wit R: Choosing chemotherapy in patients with advanced urothelial cell cancer who are unfit to receive cisplatin-based chemotherapy. Ther Adv Med Oncol 2: 381-388, 2010.

23. Dogliotti L, Carteni G, Siena S, Bertetto O, Martoni A, Bono A, Amadori D, Onat $\mathrm{H}$ and Marini L: Gemcitabine plus cisplatin versus gemcitabine plus carboplatin as first-line chemotherapy in advanced transitional cell carcinoma of the urothelium: Results of a randomized phase 2 trial. Eur Urol 52: 134-141, 2007.

24. Sekine I, Segawa Y, Kubota K and Saeki T: Risk factors of chemotherapy-induced nausea and vomiting: Index for personalized antiemetic prophylaxis. Cancer Sci 104: 711-717, 2013.

25. Mukoyama N, Yoshimi A, Goto A, Kotani H, Ishikawa K, Miyazaki N, Miyazaki M, Yamada K,Kikkawa F, Hasegawa Y, et al: An analysis of behavioral and genetic risk factors for chemotherapy-induced nausea and vomiting in Japanese subjects. Biol Pharm Bull 39: 1852-1858, 2016.

26. Ministry of Education, Culture, Sports, Science and Technology/Ministry of Health, Labor and Welfare: The ethical guidelines for medical and health research involving human subjects. https://www.mhlw.go.jp/content/000757566.pdf. Accessed March 23, 2021. (In Japanese) 